Louisiana State University

LSU Digital Commons

Faculty Publications

Department of Physics \& Astronomy

$5-1-2018$

\title{
Observation of an optical spring with a beam splitter
}

Jonathan Cripe

Louisiana State University

Baylee Danz

Brigham Young University Idaho

Benjamin Lane

Louisiana State University

Mary Catherine Lorio

Louisiana State University

Julia Falcone

Case Western Reserve University

See next page for additional authors

Follow this and additional works at: https://digitalcommons.Isu.edu/physics_astronomy_pubs

\section{Recommended Citation}

Cripe, J., Danz, B., Lane, B., Catherine Lorio, M., Falcone, J., Cole, G., \& Corbitt, T. (2018). Observation of an optical spring with a beam splitter. Optics Letters, 43 (9), 2193-2196. https://doi.org/10.1364/

OL.43.002193

This Article is brought to you for free and open access by the Department of Physics \& Astronomy at LSU Digital Commons. It has been accepted for inclusion in Faculty Publications by an authorized administrator of LSU Digital Commons. For more information, please contact ir@lsu.edu. 


\section{Authors}

Jonathan Cripe, Baylee Danz, Benjamin Lane, Mary Catherine Lorio, Julia Falcone, Garrett D. Cole, and Thomas Corbitt 


\title{
Observation of an optical spring with a beam splitter
}

\author{
Jonathan Cripe, ${ }^{1}$ Baylee Danz, ${ }^{2}$ Benjamin Lane, ${ }^{1}$ Mary Catherine Lorio, ${ }^{1}$ Julia Falcone, ${ }^{3}$ \\ Garrett D. Cole, ${ }^{4,5,6}$ (1) And Thomas Corbitt ${ }^{1, *}$ \\ ${ }^{1}$ Department of Physics \& Astronomy, Louisiana State University, Baton Rouge, Louisiana 70803, USA \\ ${ }^{2}$ Department of Physics, Brigham Young University-Idaho, Rexburg, Idaho 83460, USA \\ ${ }^{3}$ Department of Astronomy, Case Western Reserve University, Cleveland, Ohio 44106, USA \\ ${ }^{4}$ Vienna Center for Quantum Science and Technology (VCQ), Faculty of Physics, University of Vienna, A-1090 Vienna, Austria \\ ${ }^{5}$ Crystalline Mirror Solutions LLC, Santa Barbara, California 93101, USA \\ ${ }^{6}$ Crystalline Mirror Solutions GmbH, A-1060 Vienna, Austria \\ ${ }^{*}$ Corresponding author: tcorbitt@phys.Isu.edu
}

Received 9 January 2018; revised 2 April 2018; accepted 3 April 2018; posted 5 April 2018 (Doc. ID 314774); published 1 May 2018

\begin{abstract}
We present the experimental observation of an optical spring without the use of an optical cavity. The optical spring is produced by interference at a beam splitter and, in principle, does not have the damping force associated with optical springs created in detuned cavities. The experiment consists of a Michelson-Sagnac interferometer (with no recycling cavities) with a partially reflective GaAs microresonator as the beam splitter that produces the optical spring. Our experimental measurements at input powers of up to $360 \mathrm{~mW}$ show the shift of the optical spring frequency as a function of power and are in excellent agreement with theoretical predictions. In addition, we show that the optical spring is able to keep the interferometer stable and locked without the use of external feedback. () 2018 Optical Society of America
\end{abstract}

OCIS codes: (120.4880) Optomechanics; (120.3180) Interferometry; (230.3990) Micro-optical devices; (230.4910) Oscillators.

https://doi.org/10.1364/OL.43.002193

Optomechanical cavities consisting of a movable mirror or resonator allow the electromagnetic radiation of the cavity mode to couple to the motion of the mechanical oscillator. Optomechanical cavities have been proposed for improving the sensitivity of gravitational wave detectors below the standard quantum limit, tests of quantum mechanics, and quantum information [1].

One feature resulting from coupling light to a mechanical resonator in a cavity is the optical spring effect, which was first discussed for Fabry-Pérot cavities by Braginsky [2,3]. For the traditional case of the Fabry-Pérot cavity, the optical spring is created in a detuned cavity where the cavity's circulating power and, therefore, the radiation pressure force on the mirrors, is proportional to the cavity length [4]. For a blue-detuned cavity in which the cavity's resonance frequency is less than the laser frequency, the linear relationship between the radiation pressure force and cavity length creates a positive restoring force with an effective spring constant $K_{\mathrm{OS}}$ and an antidamping force $\Gamma_{\mathrm{OS}}$. The combination of the optical spring constant and the mechanical spring constant of the device shift the resonance frequency of the system from $\Omega_{m}$ to $\sqrt{\Omega_{m}^{2}+\Omega_{\text {OS }}^{2}}$ where $\Omega_{m}$ is the resonance frequency of the mechanical oscillator, and $\Omega_{\mathrm{OS}}$ is the optical spring frequency [4-6]. This frequency shift is an experimental signature of the optical spring.

The antidamping force created by the optical spring can overwhelm the mechanical damping and lead to dynamic instabilities [7-9] and is usually controlled with feedback loops [6-8]. An alternative method to stabilizing the optical spring is to modify the damping force by adding a second optical spring $[10,11]$ or utilizing thermo-optic effects $[12,13]$.

Although the detuned Fabry-Pérot cavity is the canonical example of creating an optical spring, it is possible to create an optical spring in other topologies. An optical spring can be created in any system that is able to produce a linear relationship between the radiation pressure force and displacement. Dual-recycled gravitational wave detectors such as Advanced LIGO [14] and Advanced Virgo [15] are able to create an optical spring in the signal recycling cavity by detuning the signal recycling mirror $[16,17]$. An optical spring can also be produced in a membrane in the middle setup $[18,19]$ or a Michelson-Sagnac interferometer with a tunable signal recycling mirror at the dark port $[20,21]$.

These examples, however, still rely on the use of a cavity to produce the optical spring. In this Letter, we present the measurement of an optical spring produced by the interaction of two input fields at a beam splitter, which we will refer to as the microresonator, similar to the scheme outlined in Ref. [22]. To achieve this, we utilize a Michelson-Sagnac interferometer for simplicity. Previous results using a Michelson-Sagnac interferometer have included a signal recycling mirror and have not directly observed the frequency shift that accompanies the optical spring [21]. We measure the optical spring at input powers of $50,100,200$, and $360 \mathrm{~mW}$ and compare our experimental 
results with a theoretical model. The optical springs created at all four input powers are strong enough to keep the interferometer stable and locked without the use of any external electronic feedback or additional optical fields.

The optomechanical setup is shown in Fig. 1. The in-air Michelson-Sagnac interferometer contains a partially reflective microresonator as the end/common mirror of the interferometer. The Michelson-Sagnac topology was used to simplify the alignment of the laser beams onto the microresonator. The microresonator is similar to the one used in Refs. [6,11] and described in Refs. [23,24], but consists only of a $358 \mathrm{~nm}$ thick GaAs cantilever without the highly reflective stack of crystalline $\mathrm{Al}_{0.92} \mathrm{Ga}_{0.08} \mathrm{As} / \mathrm{GaAs}$ layers. The GaAs microresonator has a power reflectivity of $R_{\mathrm{osc}}=65 \%$ for the laser wavelength of $\lambda=1064 \mathrm{~nm}$. The microresonator has a diameter of $140 \mu \mathrm{m}$, a mass of about $30 \mathrm{ng}$, a natural mechanical frequency of $\Omega_{m}=2 \pi \times 850 \mathrm{~Hz}$. The quality factors for the fundamental and yaw resonances are $Q_{m_{f}}=5$ and $Q_{m_{y}}=13$, respectively, and are obtained by matching the theoretical model to the measured data. The low mechanical Qs are the result of performing the experiment in air. A photomicrograph of the microresonator is included as a subset in Fig. 1(c).

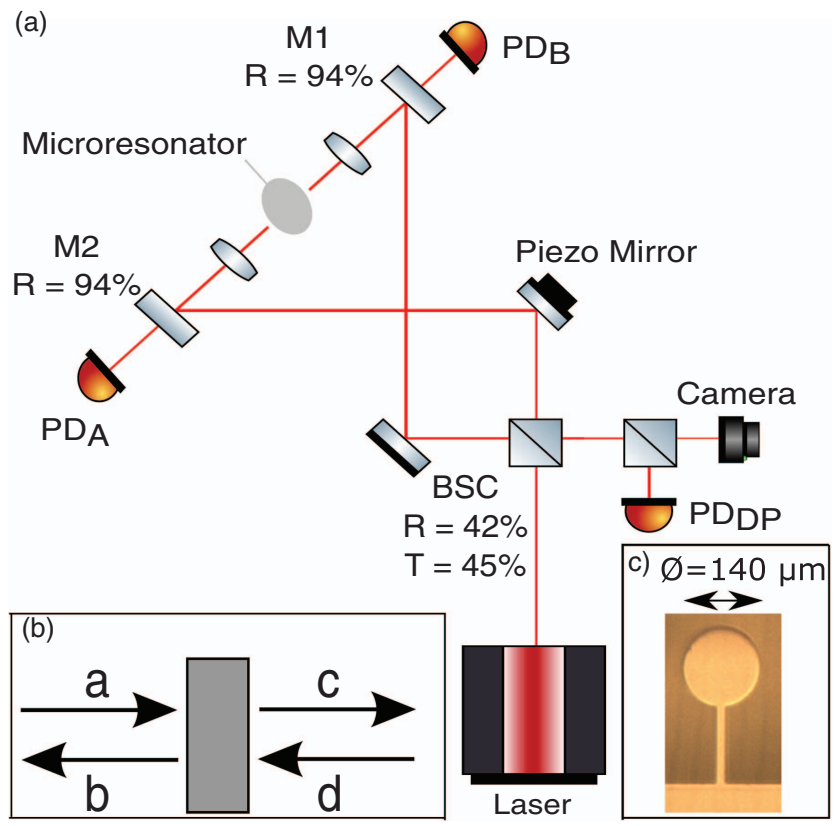

Fig. 1. Schematic of the experimental optomechanical setup. The $\mathrm{Nd}$ :YAG laser is split into two beams by a BSC and directed towards the partially reflective microresonator. One arm of the interferometer contains a steering mirror attached to a piezoelectric device that generates a phase difference $\phi$ between the two arms of the interferometer. Both arms contain partially transmissive steering mirrors (M1 and M2) that allow some of the reflected and transmitted light to be detected for locking the interferometer. The two reflected and two transmitted beams from the microresonator interfere at the BSC and are detected by a photodetector $\left(\mathrm{PD}_{\mathrm{DP}}\right)$. (b) Fields $a$ and $d$ are incident on the microresonator from opposite sides. The input fields are supplied by a laser of power $P_{0}$ and frequency $\omega_{0}$ with $a$ and $d$ each receiving about half the total power. The microresonator has power reflectivity $R_{\text {osc }}=\rho^{2}=65 \%$. (c) Photomicrograph of the microresonator with a diameter of $140 \mu \mathrm{m}$ supported by a $200 \mu \mathrm{m}$ long by $20 \mu \mathrm{m}$ wide cantilever structure.
To realize the optical spring, let us first consider the microresonator and its associated normalized fields, as shown in Fig. 1(b) [25]. Each of the normalized input fields $a$ and $d$ receives half the power from the laser source, and we allow for a phase shift in $d$, accounting for the difference between the path lengths. We assume that the motion of the microresonator is small and that the path length difference remains constant, so we relate the normalized fields:

$$
\begin{aligned}
a & =\sqrt{\frac{P_{0}}{2}}, \\
b & =\rho a+\tau d, \\
c & =\tau a-\rho d, \\
d & =\sqrt{\frac{P_{0}}{2}} e^{i \phi}, \\
\phi & =\frac{L \omega_{0}}{c},
\end{aligned}
$$

where $\rho$ and $\tau$ are the amplitude reflectivity and transmissivity such that $\rho^{2}+\tau^{2}=1, \omega_{0}$ is the laser frequency, $P_{0}$ is the laser power incident on the beam splitter cube (BSC), and $L$ and $\phi$ are the difference in length and phase of the two interferometer arms, respectively. We solve the equations and find the net power leaving the microresonator

$$
P_{\text {net }}=|b|^{2}-|c|^{2}=2 \rho \tau P_{0} \cos \phi .
$$

To understand why we are interested in the net power leaving the microresonator, consider the forces acting on the microresonator. The net force from $a, b, c$, and $d$ is

$$
F_{\text {net }}=\left(P_{a}+P_{b}-P_{c}-P_{d}\right) / c \text {. }
$$

If the input powers $P_{a}$ and $P_{d}$ are balanced, then a nonzero value for $P_{b}-P_{c}$ gives rise to a net force on the microresonator exerted by radiation pressure $F_{\mathrm{RP}}=P_{\text {net }} / c$. For small displacements $\delta L$ around an equilibrium position, the microresonator experiences the differential force

$$
\delta F_{\mathrm{RP}}=\frac{1}{c} \frac{d P_{\mathrm{net}}}{d L} \delta L,
$$

which can be expressed as the equivalent spring constant

$$
K_{\mathrm{OS}}=-\frac{1}{c} \frac{d P_{\mathrm{net}}}{d L}=\frac{2}{c^{2}} \omega P_{0} \rho \tau \sin (\phi)
$$

$\mathrm{K}_{\mathrm{OS}}$ is purely real, indicating that it provides a restoring force without the addition of a damping force that normally arises from the imaginary part of $\mathrm{K}_{\mathrm{OS}}$. While damping from the Doppler effect does exist, it is very small in comparison to the mechanical damping in the system. The maximum $\mathrm{K}_{\mathrm{OS}}$ occurs for a path difference of $\phi=\pi / 2$, as shown in Fig. 2.

One of the arms of the interferometer contains a steering mirror which is mounted onto a piezoelectric device. The piezo mirror is used to control the phase difference between the two arms of the interferometer and to lock the interferometer. The steering mirrors on either side of the microresonator have a power reflectivity of $94 \%$ to allow for some of the light to be used for locking the interferometer. The interferometer is locked by taking the signal from either $\mathrm{PD}_{\mathrm{DP}}, \mathrm{PD}_{A}$, or $\mathrm{PD}_{B}$, filtering it, and feeding it back to the piezo mounted to the mirror in one of the arms of the interferometer. The relative 


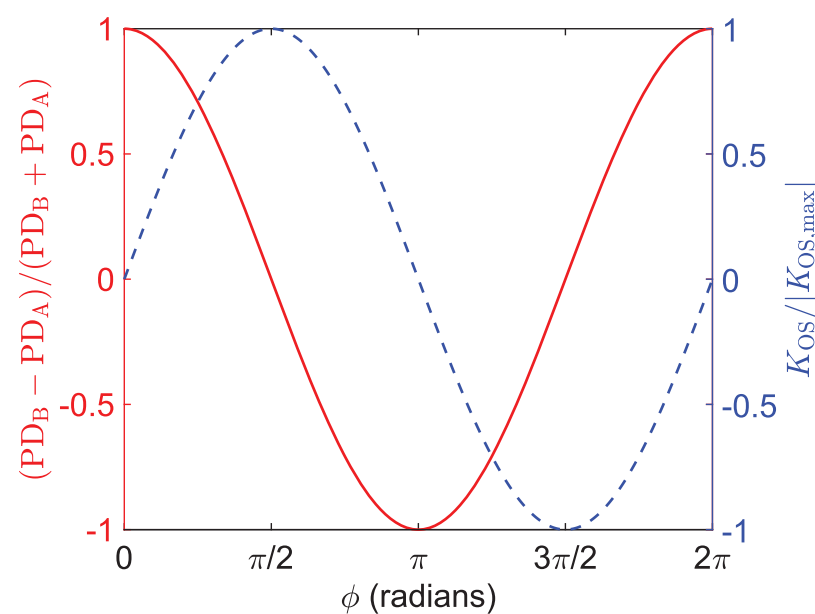

Fig. 2. Plot of the normalized power at the side photodetectors and of the normalized $\mathrm{K}_{\mathrm{OS}}$ as a function of $\phi$. The interferometer is locked at approximately $\phi=\pi / 2$, where the optical spring effect is largest.

phase difference between the two interferometer arms can be adjusted by tuning the locking setpoint on the PID controller.

We measure the optical spring effect by measuring the optical response of the system. This is performed by modulating the piezo in the interferometer and measuring the resulting power fluctuation at one of the photodetectors as a function of the modulating frequency. In the absence of an optical spring, we should measure a featureless response. However, with an optical spring, we measure the effective closed loop gain of the optomechanical system:

$$
\begin{aligned}
G_{\mathrm{cl}}= & \frac{1}{1+G_{\mathrm{OS}}}=\frac{\Omega_{m_{f}}^{2}-\Omega^{2}+i \Omega \Gamma_{m_{f}}(\Omega)}{\Omega_{m_{f}}^{2}-\Omega^{2}+i \Omega \Gamma_{m_{f}}(\Omega)+\Omega_{\mathrm{OS}}^{2}}, \\
& +\frac{\Omega_{m_{y}}^{2}-\Omega^{2}+i \Omega \Gamma_{m_{y}}}{\Omega_{m_{y}}^{2}-\Omega^{2}+i \Omega \Gamma_{m_{y}}+\Omega_{\mathrm{OS}}^{2}},
\end{aligned}
$$

as described in Ref. [6] where the first term is for the fundamental mode, and the second term is for the yaw mode with $\Gamma_{m_{f}}$ and $\Gamma_{m_{y}}$ being the mechanical damping for fundamental and yaw modes, respectively. The contribution from the yaw mode is a result of the laser beam not being perfectly centered on the microresonator. The position-dependent coupling of the optical spring to the yaw mode is analogous to attaching a mechanical spring to different points on the microresonator.

We lock the interferometer at the mid-fringe point of $\mathrm{PD}_{B}$, which corresponds to the point at which the optical spring is largest, as shown in Eqs. (6) and (9), and in Fig. 2. We measure the transfer function at input powers of 50,100,200, and $360 \mathrm{~mW}$, as shown in Fig. 3. The optical spring peak is visible in each of the measurements at frequencies of 1000, 1120, 1310 , and $1640 \mathrm{~Hz}$, as well as a dip corresponding to the fundamental mechanical resonance at about $850 \mathrm{~Hz}$. The effect of the optical spring is also visible on the yaw mode of the microresonator at $4.2 \mathrm{kHz}$.

An interesting feature of the system is its ability to remain locked without any external feedback. At all four input powers, the optical spring is strong enough to stabilize the system and keep the interferometer locked at a desired fringe setpoint

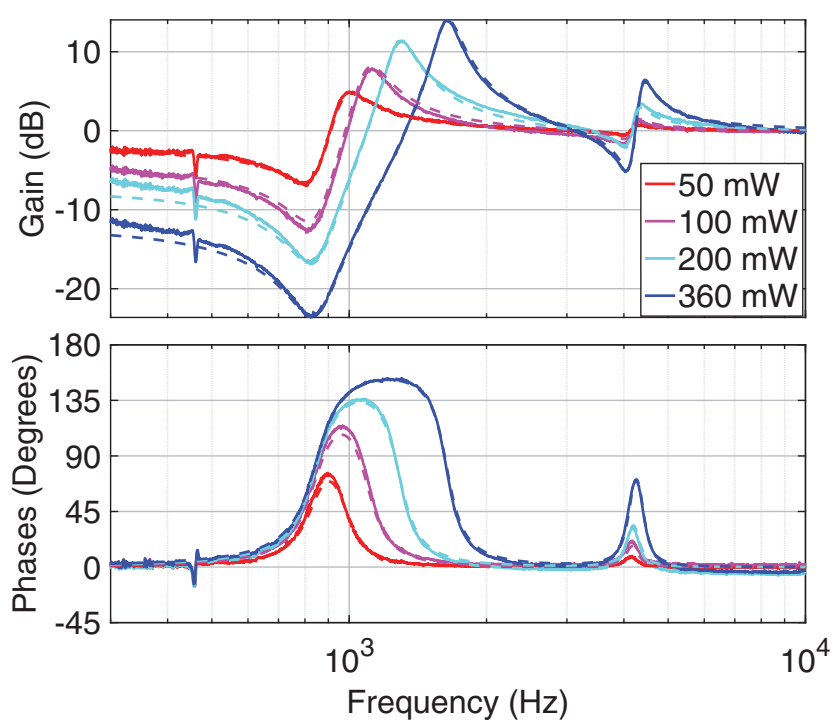

Fig. 3. Measurements (solid) and theoretical model (dashed) of the optical spring at input powers of 50,100, 200, and $360 \mathrm{~mW}$. The measured transfer function is taken by injecting a signal to the PID controller connected to the piezo mirror in one arm of the interferometer and measuring its effect at $\mathrm{PD}_{\mathrm{DP}}$. The dip at about $850 \mathrm{~Hz}$ corresponds to the fundamental mechanical resonance of the microresonator, and the feature at $4.2 \mathrm{kHz}$ is the optical spring coupled to the yaw mode of the microresonator.

without the application of any feedback. Unlike the traditional case of the optical spring in a detuned Fabry-Pérot cavity where the antidamping of the optical spring must be controlled using electronic feedback or another method, our system does not have an antidamping term and, therefore, is stable as a result of the restoring force provided by the optical spring. External disturbances at frequencies below the optical spring frequency are suppressed by a factor of approximately

$$
\frac{1}{G_{\mathrm{cl}}} \approx \frac{\Omega_{\mathrm{OS}}^{2}}{\Omega_{m}^{2}} \approx 4,
$$

for the $360 \mathrm{~mW}$ measurement at low frequencies $[5,6]$. The stability of the system is visible in Fig. 3, where the noise at $300 \mathrm{~Hz}$ is suppressed by a factor of up to $11.6 \mathrm{~dB}$ or a magnitude of approximately 4 . The measurements shown in Fig. 3 are taken with the interferometer locked with the PID controller to avoid overly exciting a resonance and causing the system to lose lock. Further suppression of the external disturbances could be achieved by increasing the optical spring frequency by increasing the input power.

In conclusion, we have shown the measurement of the optical spring from a beam splitter in a Michelson-Sagnac interferometer, without the use of a cavity. The measurements at input powers of $50,100,200$, and $360 \mathrm{~mW}$ clearly show the change in the system's resonance frequency created by the optical spring effect and match well with theoretical predictions. The optical spring created at all four input powers is strong enough to keep the interferometer stable and locked to the desired fringe setpoint and reduces disturbances at $300 \mathrm{~Hz}$ by up to $11.6 \mathrm{~dB}$.

In the future, we would like to investigate the possibility of using the partially reflective microresonators in experiments 
with quantum radiation pressure noise. As a result of not having the highly reflective stack of crystalline $\mathrm{Al}_{0.92} \mathrm{Ga}_{0.08} \mathrm{As}$ / GaAs layers, the mass of these microresonators is lower than the highly reflective microresonators. The reduction in mass, $m$, increases the signal-to-noise ratio of the quantum radiation pressure noise over the thermal noise by a factor of $\sqrt{m}$. We also aim to measure the mechanical dissipation as a function of frequency to investigate thermal noise models. In addition, the microresonators could have use in experiments studying unstable optomechanical filter cavities, such as those proposed in Ref. [26].

Funding. National Science Foundation (NSF) (PHY1150531).

\section{REFERENCES}

1. M. Aspelmeyer, T. J. Kippenberg, and F. Marquardt, Rev. Mod. Phys. 86, 1391 (2014).

2. V. B. Braginsky and I. I. Minakova, Moscow Univ. Phys. Bull. 1, 83 (1964).

3. V. B. Braginsky and A. B. Manukin, Sov. Phys. JETP 25, 653 (1967).

4. B. S. Sheard, M. B. Gray, C. M. Mow-Lowry, D. E. McClelland, and S. E. Whitcomb, Phys. Rev. A 69, 051801 (2004).

5. T. Corbitt, Y. Chen, F. Khalili, D. Ottaway, S. Vyatchanin, S. Whitcomb, and N. Mavalvala, Phys. Rev. A 73, 023801 (2006).

6. J. Cripe, N. Aggarwal, R. Singh, R. Lanza, A. Libson, M. J. Yap, G. D. Cole, D. E. McClelland, N. Mavalvala, and T. Corbitt, Phys. Rev. A 97, 013827 (2018)

7. O. Miyakawa, R. Ward, R. Adhikari, M. Evans, B. Abbott, R. Bork, D. Busby, J. Heefner, A. Ivanov, M. Smith, R. Taylor, S. Vass, A. Weinstein, M. Varvella, S. Kawamura, F. Kawazoe, S. Sakata, and C. Mow-Lowry, Phys. Rev. D 74, 022001 (2006).
8. T. Corbitt, D. Ottaway, E. Innerhofer, J. Pelc, and N. Mavalvala, Phys. Rev. A 74, 021802 (2006).

9. T. J. Kippenberg, H. Rokhsari, T. Carmon, A. Scherer, and K. J. Vahala, Phys. Rev. Lett. 95, 033901 (2005).

10. T. Corbitt, Y. Chen, E. Innerhofer, H. Müller-Ebhardt, D. Ottaway, H. Rehbein, D. Sigg, S. Whitcomb, C. Wipf, and N. Mavalvala, Phys. Rev. Lett. 98, 150802 (2007).

11. R. Singh, G. D. Cole, J. Cripe, and T. Corbitt, Phys. Rev. Lett. 117, 213604 (2016).

12. D. Kelley, J. Lough, F. Mangaña Sandoval, A. Perreca, and S. W. Ballmer, Phys. Rev. D 92, 062003 (2015).

13. P. A. Altin, T. T.-H. Nguyen, B. J. J. Slagmolen, R. L. Ward, D. A. Shaddock, and D. E. McClelland, Sci. Rep. 7, 14546 (2017).

14. LIGO Scientific Collaboration, Classical Quantum Gravity 32, 074001 (2015).

15. VIRGO Collaboration, Classical Quantum Gravity 32, 024001 (2015).

16. A. Buonanno and Y. Chen, Classical Quantum Gravity 19, 1569 (2002).

17. A. Buonanno and Y. Chen, Phys. Rev. D 65, 042001 (2002).

18. J. D. Thompson, B. M. Zwickl, A. M. Jayich, F. Marquardt, S. M. Girvin, and J. G. E. Harris, Nature 452, 72 (2008).

19. A. M. Jayich, J. C. Sankey, B. M. Zwickl, C. Yang, J. D. Thompson, S. M. Girvin, A. A. Clerk, F. Marquardt, and J. G. E. Harris, New J. Phys. 10, 095008 (2008).

20. A. Xuereb, R. Schnabel, and K. Hammerer, Phys. Rev. Lett. 107, 213604 (2011).

21. A. Sawadsky, H. Kaufer, R. M. Nia, S. P. Tarabrin, F. Y. Khalili, K. Hammerer, and R. Schnabel, Phys. Rev. Lett. 114, 043601 (2015).

22. S. L. Danilishin and F. Y. Khalili, Living Rev. Relativity 15, 5 (2012).

23. G. D. Cole, S. Gröblacher, K. Gugler, S. Gigan, and M. Aspelmeyer, Appl. Phys. Lett. 92, 261108 (2008).

24. G. D. Cole, Proc. SPIE 8458, 845807 (2012).

25. T. Corbitt, Y. Chen, and N. Mavalvala, Phys. Rev. A 72, 013818 (2005).

26. H. Miao, Y. Ma, C. Zhao, and Y. Chen, Phys. Rev. Lett. 115, 211104 (2015). 